\title{
RHESSI and TRACE observations of an M2.5 flare: a direct application of the Kopp and Pneuman model (Research Note)
}

\author{
L. Contarino ${ }^{1}$, P. Romano ${ }^{2}$, and F. Zuccarello ${ }^{1}$ \\ 1 Dipartimento di Fisica e Astronomia - Sezione Astrofisica, Università di Catania, via S. Sofia 78, 95123 Catania, Italy \\ e-mail: fzu@oact.inaf.it \\ 2 INAF - Osservatorio Astrofisico di Catania, via S. Sofia 78, 95123 Catania, Italy \\ Received 3 December 2005 / Accepted 3 July 2006
}

\section{ABSTRACT}

\begin{abstract}
Context. The processes of current sheet formation and magnetic reconnection are nowadays believed to be at the basis of several solar phenomena, including erupting prominences, flares, and CMEs. However, the possibility of determining clear signatures of the occurrence of these processes in erupting active regions is still lacking in several aspects.

Aims. In this paper we analyze coronal images relevant to a system of loops involved in a very energetic flare, to search for observational signatures of current sheet formation and magnetic reconnection and compare them with what is expected from theory. Methods. X-ray and EUV data acquired by the Ramaty High-Energy Solar Spectroscopic Imager (RHESSI) and the Transition Region and Coronal Explorer (TRACE) satellites have been used to analyze an M2.5 flare that occurred in NOAA 9901 on 16 April 2002. The evolution of the flare loops, the EUV footpoints, and the X-ray loop tops have been studied.

Results. The event shows a simple morphology in which the EUV and X-ray sources change from an $X$ to a $Y$ configuration; the formation of a thin, filamentary structure, compatible with what is expected from the collapse of an $X$-point in a current sheet, is observed; the height of the top of the observed EUV loops and the separation between the footpoints show an increase with time. Conclusions. The study of this event provides several observational signatures, expected from theory during the formation of a current sheet and consequent reconnection, which allowed us to interpret it in the framework of the Kopp and Pneuman model.
\end{abstract}

Key words. Sun: flares - Sun: activity - Sun: filaments

\section{Introduction}

Nowadays, it is widely accepted that the process of magnetic reconnection can play a fundamental role in solar flares. In about sixty years several models have been developed, from the classical reconnection models proposed by Giovannelli (1946), Hoyle (1949), Petschek (1964), Sturrock (1966), Hirayama (1974), Kopp \& Pneuman (1976), to the modern three dimensional theory (see, e.g., Priest \& Forbes 1990, 2000).

Essentially, magnetic reconnection is a topological rearrangement of the magnetic field in which the released magnetic energy is quickly converted into the thermal and kinetic energy of plasma and particles. Between oppositely directed magnetic field lines, a current sheet forms where the field lines reconnect. Despite the noticeable theoretical development, the direct observation of formation and evolution of current sheets is still lacking in several aspects.

In this regard, the possibility of observing coronal layers in EUV and $X$ ranges offered, respectively, by satellites like TRACE and RHESSI, can provide some means of verifying the above-mentioned models.

TRACE is a NASA Small Explorer mission launched in April 1998. It has a $30 \mathrm{~cm}$ telescope that uses three normal incidence coatings for EUV imagery and one for UV on corresponding quadrants of the primary and secondary mirrors. A lumigen coated $1024 \times 1024 \mathrm{CCD}$ detector collects images over an $8.5 \times 8.5$ arcmin field of view (0.5 arcsec pixels) (Tarbell et al. 1994; Handy et al. 1999).
The RHESSI spacecraft provides imaging spectroscopy in the energy range $3 \mathrm{keV}-17 \mathrm{MeV}$ (Lin et al. 2002). The instrument consists of a nine-element array of hyperpure germanium detectors viewing the Sun through a set of modulation collimators. This results in a capability for imaging spectroscopy with an angular resolution down to 2.26 arcsec (FWHM for the finest grids) and an energy resolution ranging from about $1 \mathrm{keV}$ for $\mathrm{X}$-rays to a few $\mathrm{keV}$ for $\gamma$-rays. The instrument is also capable of high time resolution, limited by counting statistics (Krucker \& Hudson 2004). Since RHESSI was launched on February 2002, it has provided many new details on solar flares (Lin et al. 2002) and current sheet formation (Sui et al. 2003).

In this work, the analysis of an energetic flare occurring in active region 9901 on 16 April 2002 and classified as a GOES M 2.5 flare is presented. To investigate the evolution of the event, simultaneous data at two coronal levels, in EUV and X-ray wavelength ranges, have been considered. The observational evidence deduced from these data has been interpreted in the framework of the classical model of magnetic reconnection proposed by Kopp \& Pneuman (1976). This model forecasts the formation of a current sheet following the collapse of an $X$-point, due to the eruption of a filament, and the energy release in a $Y$ magnetic field configuration, with the formation of a post-flare loop system that rises towards higher atmospheric layers.

\section{Data analysis}

During the M 2.5 flare that occurred in NOAA 9901 on 16 April 2002, the maximum emission in X-ray was recorded by GOES 


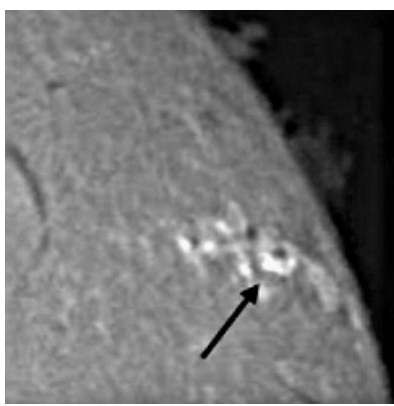

(a)

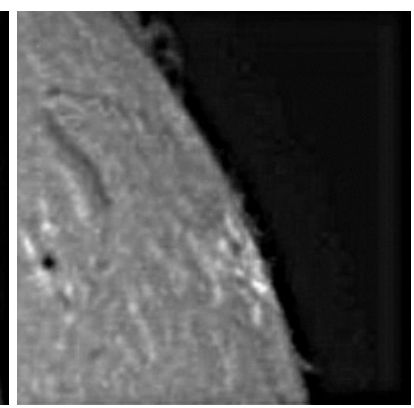

(b)

Fig. 1. $\mathrm{H}_{\alpha}$ images acquired at the INAF-Catania Astrophysical Observatory on 15 April a) and 16 April b), 2002, at 07:39 and 07:03 UT, respectively. The arrow in the image on the left indicates the presence of a filament. The field of view is $\sim 280 \times 280 \mathrm{Mm}^{2}$. North is on the top and West on the right.

at 13:18 UT (beginning and ending at 12:53 and 13:30 UT, respectively).

Figure 1 shows the region as it appears in the chromosphere in $\mathrm{H}_{\alpha}$ images acquired at the INAF-Catania Astrophysical Observatory, on 15 and 16 April, respectively. In the $\mathrm{H}_{\alpha}$ image acquired on 15 April, we can see the presence of a filament inside the region (Fig. 1a), while on 16 April, when the active region is very close to the limb $(27 \mathrm{~N}, 80 \mathrm{~W})$, the filament is not visible anymore (see Fig. 1b).

Data acquired by TRACE at $195 \AA$ and by RHESSI in the 6$12 \mathrm{keV}$ band and $12-25 \mathrm{KeV}$ band have been analyzed (because of the low count rate in the other energy bands, the relevant data are not included in this work). The images obtained at $195 \AA$ by TRACE from 12:00 to 14:59 UT cover a field of view of $768 \times$ 768 pixels $^{2}$, with a spatial and a temporal resolution of 0.5 arcsec and $\sim 1 \mathrm{~min}$, respectively. A subfield of $300 \times 300$ pixels $^{2}(105 \times$ $105 \mathrm{Mm}^{2}$ ), containing the active region under study, has been analyzed. Figure 2 shows a sequence of TRACE images of the event. About $1 \mathrm{~h}$ before the flare (12:00-13:00 UT), some very faint loops and an EUV filament channel are visible (see arrow in the first image of the sequence). During the pre-flare phase (13:07-13:17 UT) the loops increase their brightness until they reach a maximum during the flare peak (13:18 UT) and assume a cusp shape in the post-flare phase.

Moreover, the careful analysis of the TRACE image acquired at 13:07:56 UT has indicated that at this time the bright loops start to show an $X$ configuration (black arrow in the first image in Fig. 3). The $X$-configuration remains visible for several minutes before the flare peak (see the entire sequence in Fig. 3, where, to better evidence this configuration, the negative images are shown), while in the image acquired at 13:11:03 UT, a filamentary structure, indicated by the black arrow, is observed. This evolution seems to fit quite well with a scenario where a neutral magnetic $X$-point collapses in a current sheet.

A further analysis of the TRACE images has also shown that in the bright loops, during the time interval 13:10-13:40 UT, the distance between the footpoints increases. In Fig. 4 the plot of the distance between the footpoints during the analyzed time interval is reported: the distance increases from $\sim 22 \mathrm{Mm}$ to $\sim 31 \mathrm{Mm}$ with an almost linear trend. A linear fit (Fig. 4) indicates that the footpoint distance increases with a speed of about $5 \mathrm{~km} \mathrm{~s}^{-1}$.

The RHESSI X-ray light curves in the 6-12 keV band and in the 12-25 keV band show a preimpulsive burst at 13:03 UT (see the arrow in Fig. 5), as noticed by Sui et al. (2004), while

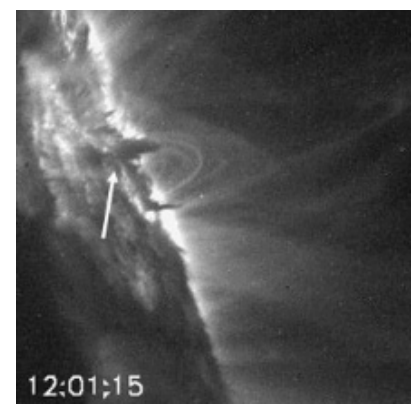

(a)

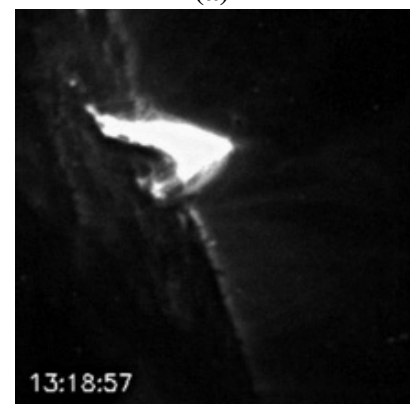

(c)

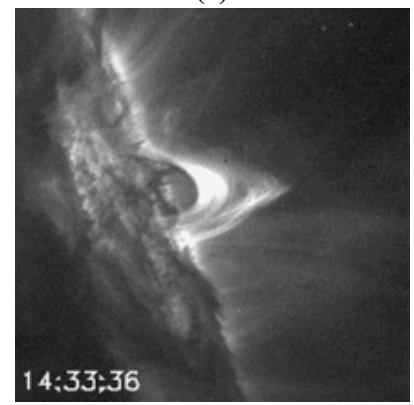

(e)

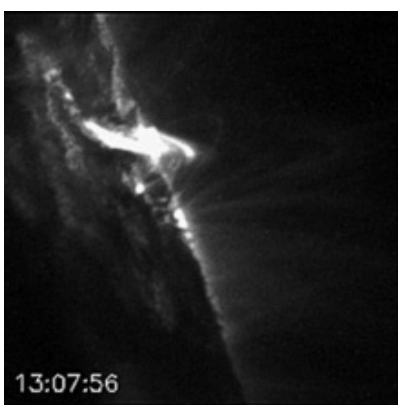

(b)

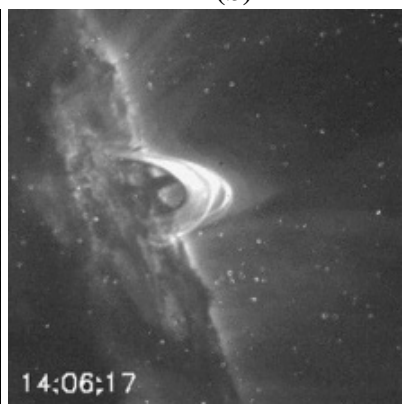

(d)

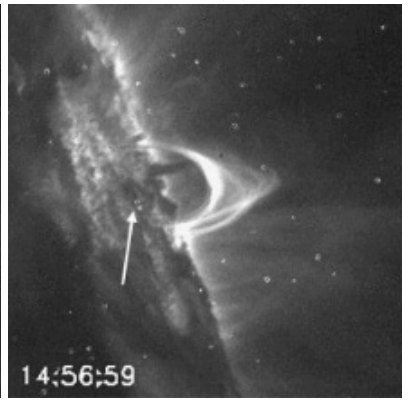

(f)
Fig. 2. Sequence of TRACE images acquired at $195 \AA$ while the loops increase their brightness and assume a cusp shape. The field of view is $\sim 105 \times 105 \mathrm{Mm}^{2}$.

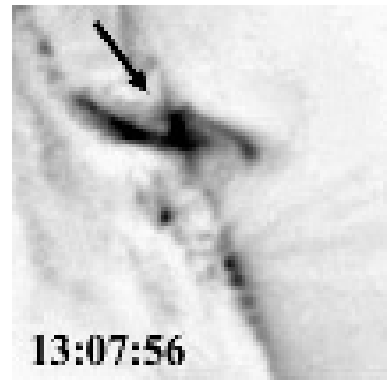

(a)

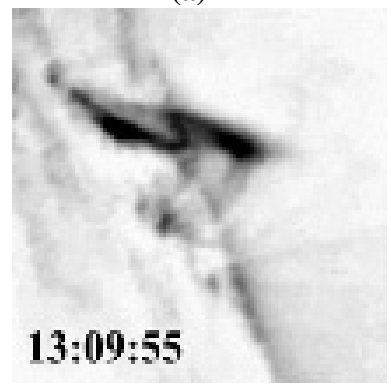

(c) (b)

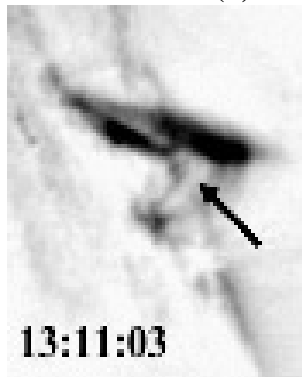

(d)

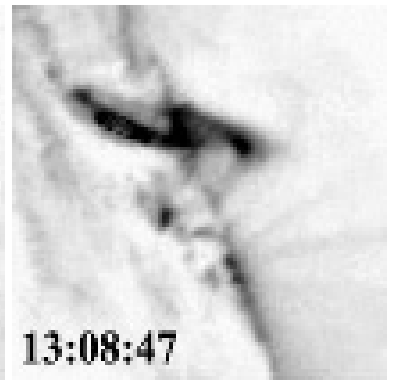

Fig. 3. Sequence of TRACE images acquired at $195 \AA$, showing the evolution of an $X$-configuration in the loops, taking place before the flare peak. The field of view is $\sim 50 \times 50 \mathrm{Mm}^{2}$. 


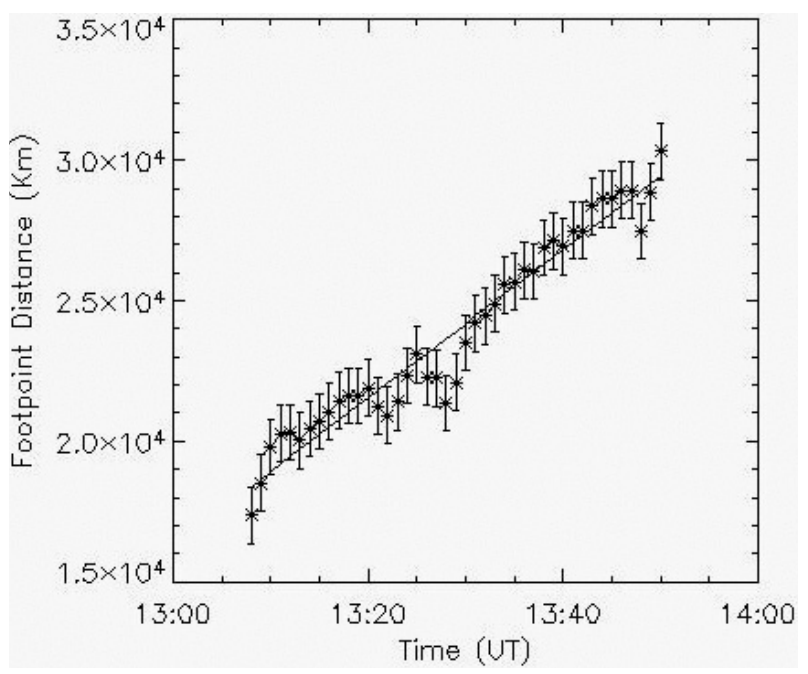

Fig. 4. Plot of the distance between the loop footpoints as a function of time. The solid line is the linear fit used to evaluate the footpoint distance growth speed.

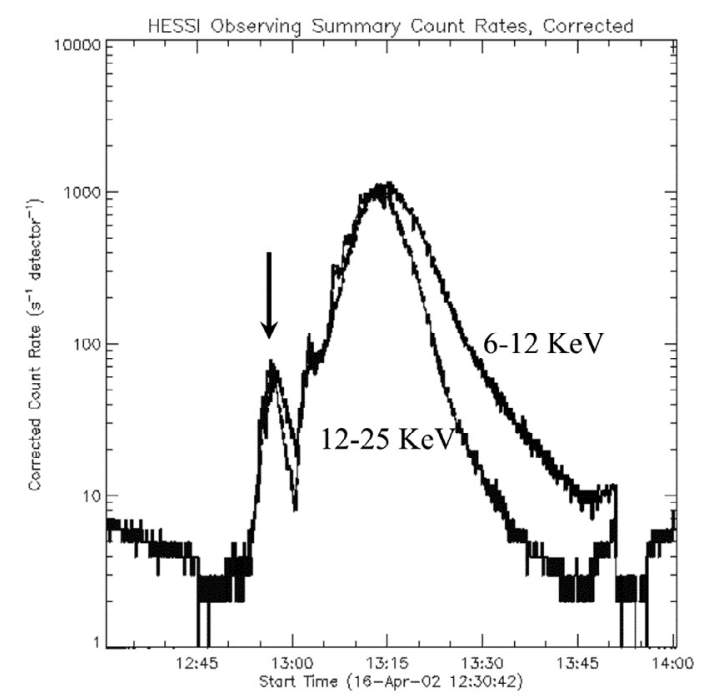

Fig. 5. RHESSI light curves of the M 2.5 flare occurred on 16 April 2002 in the $6-12 \mathrm{keV}$ and in the $12-25 \mathrm{keV}$ bands.

the impulsive phase lasts about $14 \mathrm{~min}$. In Fig. 6 we report a sequence of 12-25 keV images obtained using the CLEAN method (Hurford et al. 2002). The application of other techniques, such as back projection, MEM, MEMVIS, Pixon, and Forward-Fitting, produced similar results, characterized by the same location of the brightening during the flare peak. Moreover, using the CLEAN method we could infer, also in this wavelength range, the variation of the morphology of the possible reconnection site. More precisely, the sequence in Fig. 6 reports four images: one taken at the flare peak and three during the main phase of the flare. We have drawn the intensity iso-contour levels on RHESSI images corresponding to $13 \%$ of the peak flux in each image. The analysis of these iso-contours indicates that there is a morphological variation of the examined structure. At approximately the flare peak, the shape of the structure with thermal emission can be approximated by an $X$ configuration, while in the subsequent frames it assumes a cusp-like configuration.

Since the flare loops in the 6-12 and $12-25 \mathrm{keV}$ images have a bright loop top, we have used the centroid of the flux within the $70 \%$ contour to quantify the location of the loop top source.
Considering the loops symmetric, the height of the loop is defined as the distance between the centroid and solar limb, whose position is obtained from the recent measures of the solar radius with RHESSI (Fivian et al. 2005). In Figs. 7a and 7b we report the behavior of the height of the loop top source from the solar limb as a function of time in the $6-12 \mathrm{keV}$ band and in the 12-25 keV band, respectively. In both energy bands we found a continuous increase of height with time, with a steeper behavior from 13:03 UT to 13:07 UT, i.e., about ten minutes before the flare peak. The negative value of the height of the loop top source during the first phase of the flare is due to the projection effect because the NOAA 9901 is not perfectly at the solar limb, as we can see from the first two images in Fig. 2. At the end of the main phase, the loop height increases to $1.5 \times 10^{4} \mathrm{~km}$. Therefore, the loop growth speed is about $17 \mathrm{~km} \mathrm{~s}^{-1}$. This value is in agreement with that estimated in the Kopp \& Pneuman model $\left(\sim 20 \mathrm{~km} \mathrm{~s}^{-1}\right)$. From the comparison of both plots, we deduce that the $12-25 \mathrm{keV}$ source is higher than the 6-12 kev one during the entire flare time interval. This pattern of the loop top is consistent with the standard model of sequential magnetic reconnection onto longer and longer field lines as the reconnection point rises in the corona at higher and higher temperatures (Krucker \& Hudson 2004).

\section{Discussion and conclusions}

The analysis of X-ray and EUV data allowed us to study the temporal and spatial evolution both of the loop top source and the EUV footpoints and compare the results with a reconnection model. The results obtained can be summarized in the following main points:

- TRACE EUV images acquired during the pre-flare phase initially show the presence of an $X$ configuration and, after few minutes, a filamentary feature, apparently connecting the lower and the higher loops, which resembles the formation of a vertical current sheet following the collapse of an $X$ neutral point.

- RHESSI data indicate a change of the top-loop X-ray emission source from an $X$ configuration, during the pre-flare phase, to a $Y$ configuration, after the flare peak. The loop structures assume the canonical cusp-shape foreseen in several solar flare models.

- The centroid of the X-ray emission, assumed to be coincident with the top of the loop, shows a continuous increase of height with time, with a steeper increase ten minutes before the flare peak. The loop growth speed is about $17 \mathrm{~km} \mathrm{~s}^{-1}$, in agreement with the Kopp-Pneuman model; moreover, the $12-25 \mathrm{keV}$ source is always higher than the $6-12 \mathrm{keV}$ one.

- The separation between EUV loop footpoints increases during the flare with a linear trend and a growth speed of about $5 \mathrm{~km} \mathrm{~s}^{-1}$.

On the basis of these results, the event can be interpreted in the framework of the model of Kopp \& Pneuman (1976). The model forecasts the storage of energy in a force-free arcade supporting a filament. The eruption of the filament breaks the magnetic field lines; a neutral current sheet forms, and by means of magnetic reconnection the magnetic energy is converted into kinetic and thermal energies. The observational consequences of this process are flare loops apparently rising in the corona and the formation of two bright ribbons in the inner layers of the solar atmosphere. The motion of the loops and ribbons are not due to effective mass motion of the plasma, but to the propagation of 


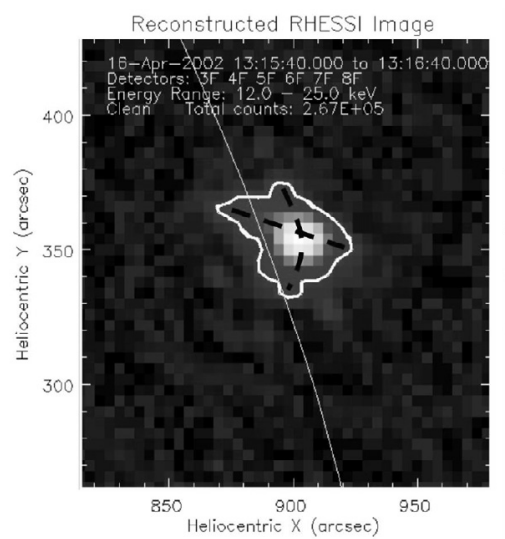

(a)

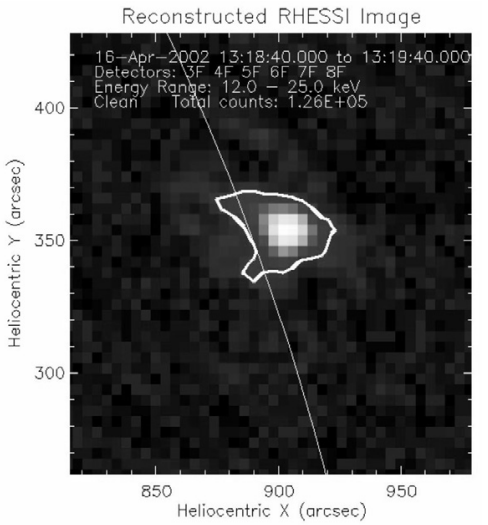

(c)

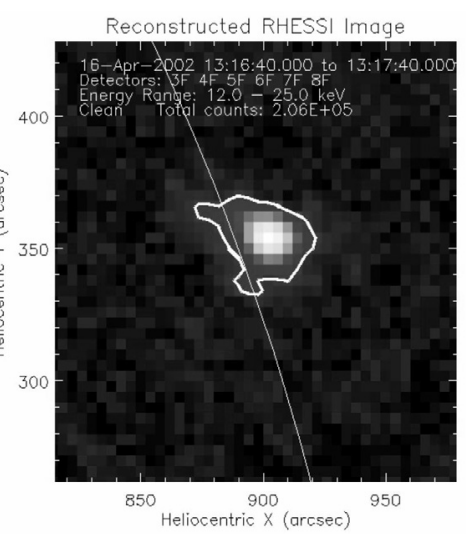

(b)

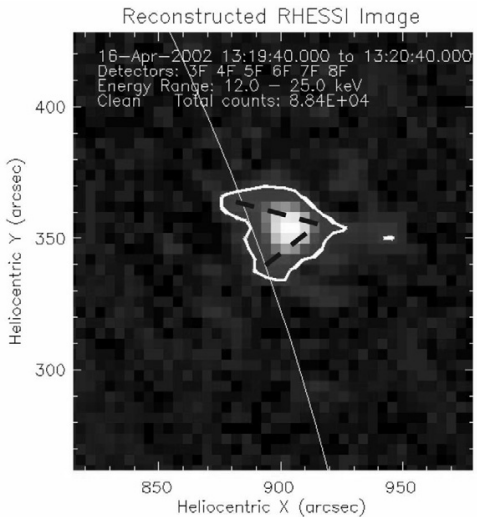

(d)

Fig. 6. Sequence of RHESSI images analyzed with the CLEAN method. The dotted lines show the shape of the structure. It has an $X$ configuration in the pre-flare phase and a $Y$ configuration after the flare peak.

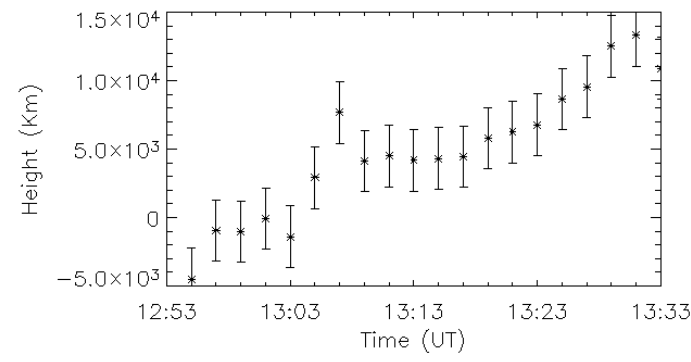

(a)

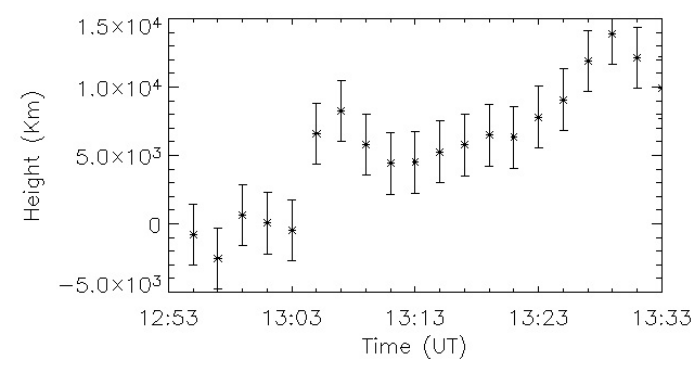

(b)

Fig. 7. Plots of the loop height versus time a) in the $6-12 \mathrm{keV}$ band and b) in the $12-25 \mathrm{keV}$ band.

the process at magnetic field lines at higher altitudes (Schmieder et al. 1987). The images we have analyzed provide an indication that the flare is triggered by the eruption of the filament initially present in the region (see Fig. 1a).
Acknowledgements. The authors wish to thank E. Catinoto, P. Costa, and S. Sciuto for their kind support and cooperation in getting $\mathrm{H} \alpha$ images at the INAFCatania Astrophysical Observatory. The authors also wish to thank the RHESSI and TRACE teams for their open data policy and the free diffusion of the software for the preliminary data analysis. This work was supported in part by the Italian Ministry of Education, University and Research, in part by the Istituto Nazionale di Astrofisica (INAF), in part by the Agenzia Spaziale Italiana (contract I/035/0 ASI/INAF-OATO), and in part by the Università degli Studi di Catania.

\section{References}

Fivian, M. D., Hudson, H. S., \& Lin, R. P. 2005, Proc. of the 11th European Solar Physics Meeting, ESA SP-600, 4

Giovanelli, R. G. 1946, Nature, 158, 81

Handy, B. N., Acton, L. W., Kankelborg, C. C., et al. 1999, Sol. Phys., 187, 229 Hirayama, T. 1974, Sol. Phys., 34, 323

Hoyle, F. 1949, Some Recent Researches in Solar Physics (Cambridge University Press)

Koop, R. A., \& Pneuman, G. W. 1976, Sol. Phys., 50, 85

Krucker, S., \& Hudson, H. S. 2004, Proc. of the SOHO 15 Workshop, ESA SP-575, 247

Lin, R. P., Dennis, B. R., Hurford, G. Y., et al. 2002, Sol. Phys., 210, 3

Petschek, H. E. 1964, Physics of Solar Flares, ed. W. N. Hess, NASA SP-50, 425 Priest, E. R., \& Forbes, T. G. 1990, Sol. Phys., 126, 319

Priest, E. R., \& Forbes, T. G. 2000, Magnetic Reconnection: MHD Theory and Applications (Cambridge University Press)

Schmieder, B., Forbes, T., Malherbe, J. M., \& Machado, M. E. 1987, ApJ, 317, 956

Sturrock, P. A. 1980, Solar Flares - A Monograph from Skylab Solar Workshop II (Boulder: Colorado Associated University Press)

Sui, L., \& Holman, G. D. 2003, ApJ, 596, L251

Sui, L., Holman, G. D. \& Dennis, B. R. 2004, ApJ, 612, 546

Tarbell, T. D., Dennis, B. R., Hurford, G. Y., et al. 1994, Proc. 3d SOHO Workshop, Solar Dynamic Phenomena and Solar Wind Consequences, ESA SP-373 (Paris: ESA), 375 